\title{
Keys to Building a Safe and Effective Healthcare Laser Program
}

\author{
By: Penny J. Smalley, RN, CMLSO \\ Technology Concepts International, Chicago, Illinois, USA
}

Laser safety is much more than wearing safety goggles, and posting a sign on the treatment room door. Compliance audits from facilities in a number of different countries, have revealed misconceptions, lack of education and training, areas of non-compliance with standards, and potentially unsafe practices that could result in staff and patient injuries. Far too often, laser safety is underappreciated and poorly implemented.

\begin{abstract}
A laser is as safe or as hazardous as the user. The knowledge and skill of the user and members of the surgical team, and the level of administrative support allocated to laser program management, will determine how well and safely a clinical practice operates. Healthcare lasers have the same risks and hazards, regardless of where they are used. Therefore, standards and guidelines for safety should be the same for all practice settings, including hospitals, clinics, or private office facilities.
\end{abstract}

Of all hazards, complacency is the most dangerous. Too often, what begins as a commitment to safety fades over time. The best way to combat apathy, and maintain a level of compliance and attention to safe practice, is to establish a laser program on a foundation of risk management principles and evidence based standards.

Proper safety management requires a fourfold approach including: initial and ongoing education and training, identification of hazards and risks, consistent use of appropriate control measures, and sustainable program audit and management.

\section{It is time to rethink laser safety.}

Safety is a system, made up of many parts, including equipment, technical skill, cognitive skills, education, competency training, collaborative practice, knowledge of standards, regulations, policies, and guidelines, and commitment to best practices. One of the most highly respected, and applicable models for establishing a culture of safety, is through the application of the principles of what is known as "Crew Resource Management (CRM)" CRM is a leadership and management system that has been proven to minimize stress, reduce error, improve safety, and enhance efficiency. It has become the gold standard in the airline industry for enhancing the way professional teams work together, and is easily applicable in surgery.

Flight crews and surgical teams are similar in many ways. Both are made up of different types of professionals working together in a confined, high pressure, high risk working environment, with the singular goal of providing a safe outcome. Pilots and surgeons have similar responsibilities for the lives and safety of those in their care, co-pilots work under the same rules as do surgical assistants or registrars, ready to step in as needed, and flight crews work under the same organizational hierarchy as staff in the operating room, with direct care of the passenger/patient, and under the supervision of the pilot/surgeon.

When CRM is applied in the operating room, surgical teams may be better able to maintain situational awareness, collaborative practice, and rapid, appropriate response to hazardous situations. CRM addresses the pitfalls in communications including: faulty processing of information, failure of team members to share both success and failure, the impact of human factors that lead to error, such as fatigue, stress, workload, lack of preparation or knowledge, and difficult interpersonal relationships.

When surgical teams follow the CRM model, individuals work together through a systematic decision making process where everyone shares responsibility, everyone is empowered to speak up and advocate for what they know to be correct practice, and every team member's voice is heard and respected. The principles of CRM are designed to replace individual emotional response with fact based response by collectively:

- gathering all available information and evidence

- evaluating the quality of the information (is it unbiased, unemotional, appropriate to the situation, evidence based and possible to accomplish ? etc.)

- determining desired and alternative courses of action 
- executing the best or chosen course of action

- objectively reviewing results

- applying lessons learned to similar events in the future

When considering a surgical team's reaction to a laser hazard during surgery, CRM might be applied by enabling nurses and doctors to work together, without pressure or blame toward the best possible outcome, thereby protecting themselves and their patients from harm.

An example of shared decision making:

A nurse notices that the drapes surrounding the $\mathrm{CO}_{2}$ laser surgical site, are dry and could present a flammability hazard if struck by a direct or indirect laser beam. She asks the surgeon to wet the drape before activating the laser. Without the CRM process, this suggestion may be overlooked or not acknowledged in the interest of time, putting everyone in the room including the patient, at risk for fire in the OR, and burns. With the CRM process in place, the surgeon hears the nurse, acknowledges her suggestion and they can then evaluate the situation based on prevailing information, policy and standards. This might include reviewing the flammability warning on the drape package and the hospital policy on preventing surgical fires, remembering their education regarding the characteristics of laser energy and tissue interaction, and relying on their experience in using the device. They then apply that information and decide that the correct course of action is to wet the drapes surrounding the site, and then ensure that the drapes remain wet, for the duration of the case.

\section{Second example:}

An anaesthetist prepares to intubate a patient with a standard PVC endotracheal tube, in preparation for a $\mathrm{CO}_{2}$ laser ablation of vocal cord nodules, using a microscope focused through the laryngoscope. The nurse in the room observes this as she is setting up the laser equipment, and based on her knowledge of the airway management protocol in the hospital, she asks him to stop, and replace the PVC tube with the proper ET tube that is approved for $\mathrm{CO}_{2}$ laser procedures in the airway. The anaesthetist may be annoyed and might tend to dismiss the nurse's suggestion if not for training in CRM. Following the CRM model, the nurse presents the shared airway protocol, and they both review the section on selection of proper ET tubes for $\mathrm{CO}_{2}$ laser cases. To save time and stress, the nurse then offers to go and get the proper tube. Without this cooperative problem solving approach, the patient is put in a situation of critical risk from airway fire, which is known to result in a high degree of morbidity and mortality.

Collaborative practice and problem solving can ease tensions in the OR, establish the best response to hazards, and protect the patient from at risk situations and potential harm. This approach should be adopted by every laser service in every healthcare facility.

\section{Remember - Laser Safety is everyone’s concern !!}

In developing a laser safety program, the first question to be asked is, "What standards and policies apply ?"

\section{Step One: Build a Foundation Based On Situational Awareness, Risk Assessment, And Knowledge Of Stan- dards, Regulations, And Best Practice Guidelines}

Standards are generally non-regulatory, but serve as consensus documents for best practice. As such, they are often considered as the usual and customary practice in a given area, and are the basis for local rules, accreditation, and medical-legal decisions in cases of patient or staff injury, incident, accident, or unanticipated occurrence. Many countries have their own national standards, which contain requirements for compliance in acquisition, installation, testing, maintenance, operation and use, of healthcare lasers. If no national standards exist, facilities can use the guidance found in the International Electrotechnical Commission (IEC) and International Standards Organization (ISO) laser standards.

Regardless of whether or not a country has national standards, laser safety requirements are generally included in prevailing Occupational Health and Safety regulations. Most of these are similar in content and guiding principles, and include a clearly defined duty of care between facility and healthcare providers. They also contain a statement that in essence, requires facilities (or owners) to mitigate all known hazards identified in the workplace. Therefore, since laser hazards are workplace hazards (operating rooms are healthcare workplaces) they would come under the jurisdic- 
tion of Health and Safety regulations. In terms of laser safety, these known hazards may include but are not limited to: possible ocular exposure and injury, flammability and reflectivity hazards, exposure to airborne contaminants in surgical plume, inadvertent exposure to the beam, and electrical hazards.

All standards address hazard analysis, risk assessment and management, administrative control measures, engineering control measures, and procedural control measures. Because standards provide the foundation for safety, surgical team members must understand their responsibilities relative to the requirements in the standards. Therefore, it is necessary to know the meaning of the key words used.

\section{Material in the standard is either informative or normative.}

Informative sections include: appendices, narrative clinical application, sample policies and documentation forms, and references, but do not specify requirements for compliance, and do not contain the key words SHOULD or SHALL.

Normative sections contain the mandatory requirements for compliance. The terms Should and Shall are found throughout the normative part of the standard. The term should means that material is strongly recommended but not necessarily mandatory, if the user has a rationale when opting for non-compliance. Because standards represent best practice, compliance is strongly recommended, but remains at the discretion of the Laser Safety Officer (LSO), for interpretation and implementation.

The term shall means that material is mandatory for compliance. Only normative material contains shalls. Where there is a NOTE:, following a normative statement, the text is intended as further explanation or support for the statement, but it cannot contain shalls.

Requirements in the standards, are assigned to the person who is responsible. The two terms defining these roles are OPERATOR, and USER. The operator is the person who manages, sets up and tests the equipment, and runs the control panel during surgery. He or she must monitor safety in the laser room throughout the surgical procedure, and ensure good communications amongst team members. This person must have the education and training to know how to identify a hazard, and what the proper response should be. If utilising CRM, the operator must be empowered to act quickly to prevent risk of injury or incident.

The user is the person who is responsible for delivering the laser energy to the indicated target. This may be the surgeon as he or she performs a procedure, the biomedical engineer during service or maintenance, a salesperson during a demonstration, or a nurse during pre-operative testing of the laser equipment. The user's main responsibility is to control the energy, apply it as intended in the safest way possible, and to have the knowledge, skill, and credentials to work within the bounds of good surgical practice, and compliance with safety requirements.

All safety standards include the mandate for education and training, and recommend the scope of curriculum and implementation. Education should be non-commercial and presented in the classroom through a curriculum that includes theory, laser science, and clinical evidence. It should be presented at a professional level, by qualified, experienced instructors, and validated by exam or other mechanism for measurement of acquired knowledge.

Surgical team members - nurses included - cannot assess risk and therefore keep themselves or their patients safe, if they do not understand the science first.

Basic education should include:

- how laser light is produced and why it differs from ordinary light,

- beam characteristics and parametres ((spot size, power density, energy density, pulsed vs continuous wave, TEM00 mode and how to assess it, test firing process, etc.)

- tissue interactions (selective photothermolysis, vaporization vs. coagulation, water transmission and absorption, etc.) - delivery systems and how to use them to optimize treatment outcomes

Manufacturers generally do not provide professional levels of education, but do have the responsibility for operational training for both nurses and surgeons. Training should be required of all users and operators, be competency based 
and validated by physical demonstration of proficiency. This should include:

- correct way to operate the controls (meaning of all symbols and words on the console)

- handling, set up and positioning of the equipment

- cleaning and daily maintenance of all equipment and accessories

- pre-operative testing

- handling of delivery systems (handpieces, micromanipulators, scanners, fibres)

Both education and training are needed to prepare healthcare professionals for the safe and effective use of lasers in surgery. This preparation must be provided to all team members, before they are expected to begin patient procedures, and must not be offered independently of one another. Training should be done only after competent, professional education is completed.

A copy of the facility's policies and procedures, and the applicable standards and guidelines, should be kept in every area of the facility where lasers are used (OR, clinics, physio, interventional radiology, etc.), for easy reference

$$
\text { Remember }- \text { Staff safety first = patient safety always ! }
$$

Second question to be asked: "What can go wrong?"

\section{Step Two: Identification of Hazards and Assessment of Risks}

Not all laser safety requirements apply to every laser. Each wavelength, system, delivery device, and application, must be assessed for applicable hazards, since they are all different, and will require different management and procedures. It is not appropriate to apply standards for a $\mathrm{CO}_{2}$ laser used in surgery, to a visible wavelength diode laser used for photobiomodulation. Knowing the science, the tissue effects, and the applications, underpins the overall understanding of what occurs when a laser is switched on, and what hazards may be present during each phase of it's use. Once these properties are well understood, the clinician can anticipate specific hazards.

Hazards are those potentially dangerous conditions associated with an unanticipated exposure. In categorizing laser hazards, we think of what happens when a laser beam interacts with tissues or materials, and how the laser device is to be used.

These hazards include: burns, eye damage, endotracheal tube fire, drape fire, explosion of gases, or non-beam hazards (those that are secondary to the actual beam interaction) such as laser generated airborne contaminants, electrical damage, toxic dyes, traffic in and out of the room, poorly trained staff, interruptions, and system failures.

Once the hazards are identified, risk must be assessed. Risk can be defined as the potential for a hazard to cause harm, and it's level of severity to those exposed.

Consider the variables when assessing the level of RISK :

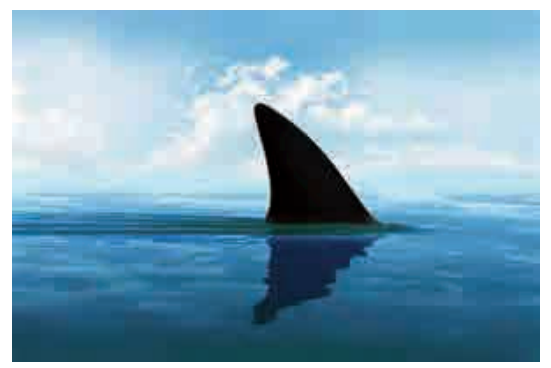

The hazard is obvious.

How does the risk vary if:

- You are on a cruise ship

- In a kayak

- In the water
Safety measures vary with levels of risk

Using this approach, consider the variables that affect risk of exposure to laser hazards. These may include but are not limited to:

- what laser you are going to use

- delivery system / instrumentation and accessory devices

- positioning of patient, draping and prepping materials

- surgeon's techniques and preferences 
- anaesthetist's access to head and neck

- number of staff in the room

- training and experience of all personnel in the room

- presence of ancillary persons

Each of these questions helps assess what control measures need to be in place. Additionally, risk levels may differ for each member of the laser team, and for each person involved with the laser equipment. While everyone in the laser treatment room has the risk of eye exposure and damage if they are unprotected, there are going to be varied risks for physician, assistant, nurse practitioner, patient, patient support person, technician, office manager, laser safety officer, scrub nurse, sales representative, biomedical engineer, and manager. Therefore, the LSO must understand each person's level of interaction with the system, and their job responsibilities, before developing appropriate policies and procedures.

Should a laser be assessed to have minimal hazards, the LSO may modify control measures to reflect those specific hazards. This is especially true for photobiomodulation laser practices. Because of variables, it is essential for each facility to develop individualized risk based policies and procedures, and not simply adopt generic documents obtained from manufacturers, course materials, or other institutions.

When a laser is purchased the hospital or clinic becomes the "Responsible Person" as defined in IEC standards (IEC 60825), and must ensure that several administrative controls are in place before personnel operate the laser, perform clinical procedures, or even test or practice with the laser.

The Responsible Person must:

- appoint a Laser Safety Officer with authority to manage and oversee all laser use

- evaluate the hazards and risks associated with the device to be purchased,

- write appropriate policies and procedures based on the risk assessment

- ensure that all surgical team members have completed laser education - training is done when the laser has been commissioned for use in the facility

- establish a mechanism for maintenance, repair, and service, according to local rules

- approved an on-going and sustainable audit / quality process

- developed documentation tools as required by the facility and by standards

Remember - Commitment to compliance is the key to all of laser safety.

"Unless commitment is made, there are only promises and hopes; but no plans."

(Peter F. Drucker)

Next Question to be asked, "How do we go about ensuring safe practice?"

Risk Management Step Three: Implementation and Monitoring of Control Measures

Control measures are the actions taken by healthcare personnel, to prevent injury or exposure to identified hazards, and are part of Policy and procedure. Policy contains clear statements of scope (who does the policy affect), rationale (why is it necessary), who is responsible for implementation and enforcement, and how compliance should be monitored.

Operational procedures are steps taken in order to comply with the policy, and are called control measures, in standards. Control measures are prescribed by facility policy, and are based on the hazards identified during risk assessment. Policies and procedures should be reviewed and if needed updated; on an annual basis, when new equipment or clinical applications are introduced, if a new surgeon arrives and is credentialed to use the laser, and whenever a new regulation or standard is published. It is the responsibility of the LSO to enforce compliance with all control measures.

There are three kinds of controls measures:

1. Engineering controls are devices designed for the specific control of a known hazard, or a feature that is built into the laser system to provide safety. They are supplied by the manufacturer in compliance with IEC standards, and 
should not be modified or defeated by the laser owner. These include guarded footswitch, audible and visible emission indicators, stand-by control, emergency off button, housing interlocks, beam attenuators. and surgical plume evacuation systems, and manual shutter blockers.

2. Procedural controls, are operational activities, specific to equipment and practice, and may include: ocular protection, fire prevention, controlled access, management of surgical plume, control of electrical hazards, control of the delivery system and beam reflectivity.

3. Administrative controls are the infrastructure of the laser safety program, and should include all requirements designated as shalls in the standards and by local rules:
a. Appointment of a Laser Safety Officer (LSO) and possible DLSO (deputy LSO)
b. Convening a laser safety committee (LSC)
c. Approval of medical credentialing criteria and nursing certification
d. Documentation tools
e. Audit and quality process in place
f. Education and training plans initiated

\section{Procedural Control Measures}

Common hazards in the laser treatment room can be mitigated by adhering to policies and procedures for: controlled access to the room and to the equipment, proper selection and use of laser protective eyewear (LPE), proper testing and monitoring of the laser and it's delivery systems throughout use, appropriate clinical application, ensuring only properly educated and trained personnel are working in the laser room, vigilance on the part of each laser team member, and using the communication and decision making tools described by CRM.

\section{Controlled Access}

Standards indicate procedures for maintaining a controlled access area. Some of the key points are:

1. Regulation warning (DANGER) signs are posted visibly, on each entryway into the laser room.

2. Appropriate protective eyewear properly labeled with the wavelength of laser in use, and required optical density, is placed with the signs at each entryway. These are removed only at conclusion of the procedure.

3. Windows are covered with approved, non-flammable barriers that meet infection control rules, are labeled for wavelength and optical density according to IEC standards, and that have been tested by a professional and reliable source to reduce transmission of the beam to acceptable levels below the hazard threshold. Laser wavelengths that absorb in water, such as $\mathrm{CO}_{2}$, do not need window barrier protection.

4. Doors are kept closed but not locked, at all times during laser use.

5. Keys are not left in the control panel of the laser and are removed and stored in a manner allowing only authorized personnel to access them when needed.

6. Positioning a dedicated operator at the control panel whenever the laser is in use. Lasers should never be left activated and unattended. If the operator has to leave the room, the laser should be turned off, and the key removed and either stored, or taken with the operator or LSO. If the operator sets up and tests the laser, but then must wait while the patient comes into the room, the laser must be kept in stand-by. This mode deactivates the shutter, and prevents accidental misfiring. The only time the laser should be turned to the ready mode, is when the clinician is aimed at target tissue, and is ready to treat.

\section{IEC Hazard Classification of lasers determines which control measures apply.}

Safety precautions, including eye protection, flammability, reflection, and administrative control measures, are determined by the classification of the laser, which must be included by the manufacturer on the device and aperture labels. Current classifications have been adopted by the IEC as follows:

Class 1: safe under every conceivable condition of use, and are, therefore, exempt from control measures 
Class $1 \mathrm{C}$ : laser radiation that is to be applied in contact with the target and has safeguards that prevent leakage of laser radiation in excess of Class 1 . It must comply with IEC 60825-1, Edition 3.0, 2014. NOTE-Typical Class $1 \mathrm{C}$ laser products include hair removal, wrinkle and acne reduction,or those for home-use.

Class 1M: Not hazardous, unless viewed with an optical instrument such as an eye-loupe or a telescope, and exempt from control measures other than to prevent optically aided viewing.

Class 2 : Visible wavelengths (400-700nm) safe if viewed for less than 0.25 seconds

2M: Visible wavelengths (400-700nm) not safe with optical viewing aids

Class 3R: Low-risk for injury with reduced safety requirements. Eye injury can occur under some direct and specular viewing conditions.

Class 3B: Can be hazardous to the eye injury from direct but not necessarily diffuse energy, and is not normally considered a skin or fire hazard.

Class 4: High power energy that is hazardous to skin and eye from direct and diffuse reflection, is a fire hazard, and can produce airborne contaminants and plasma radiation

Most all medical and surgical lasers are Class $3 b$ and Class 4 and should NEVER be operated without properly selected and fitted protective eyewear.

The classification of the laser is based on whether or not the Maximum Permissible Emission (MPE) is longer or shorter than the human aversion response. MPE is a calculation that determines how long an unprotected eye can be exposed to a laser beam before injury occurs. The aversion response is that autonomic response (within .25seconds) of the eye moving away from an intense light.

The lower classifications $(1,2)$ have extended MPE measurements, and do not require protective eyewear, since the human eye will avert from the bright light long before the beam can injure the unprotected eye.

In the case of a higher classification (3b-4) the MPE is shorter than the aversion response, and therefore, protective eyewear must be worn at all times during laser activation. Low power lasers (e.g. those used in physiotherapy), are usually lower classifications, and the LSO must determine what control measures are appropriate.

\section{Ocular Hazards}

The types of ocular injuries that can occur are determined by the interaction of the beam with the exposed tissue, and what absorption chromophores are present. Delivery systems, power and energy density, and clinical application techniques also contribute to the type and severity of damage that can occur.

$\mathrm{CO}_{2}$ and Er:YAG (long infrared wavelengths) are absorbed by water in the tissues, and therefore, can absorb at the tear layer that lubricates the cornea, and then the corneal tissue, causing burns. This is not permanent but can be painful and temporarily disabling.

Mid range infrared (Ho:YAG) can partially absorb, and partially transmit through water, and can cause corneal burns and has the potential to be absorbed at the lens, causing injury to the lens. This injury may be serious, but it is not permanently damaging to vision.

Short wavelengths (near infrared through visible range), penetrate through water, and can transmit through all anterior structures of the eye, absorbing in haemoglobin in the retina, causing permanent damage to central vision. Furthermore, the human lens acts to cause convergence of stray, low power, reflected or scattered beam emissions, which can increase power density to a significant level, and increased severity of damage to the retina.

Criteria as follows should be used to select eyewear with emphasis on the fact that all eyewear must be approved by the LSO.

1. Readable permanent labels stating wavelength in nanometers or microns and optical density (OD) appropriate 
for the laser to be used

2. Side shield protection

3. Adequate visible light transmission

4. Resist shock, scratching, and front surface reflection

5. Have proper fit and be comfortable (no slippage)

6. Intact lenses and frames free of damage, scratches, cracks, or discoloration

NEVER use splash glasses, prescription eyewear, face shields, tanning booth goggles, contact lenses, swim goggles, foil masks, or any other device not specifically designed, tested, and labeled, for laser safety use.

\section{Regardless of routine safety inspections by the LSO, each individual must ensure that the glasses he or she uses are the right ones, and that they are in safe condition, every time they are used.}

Safety eyewear is the best method of providing patient eye protection. Straps or elastic bands should be in place to keep the goggles from slipping out of place when the patient moves or is repositioned. If unable to wear goggles, due to treatment in the periorbital area, metal corneal eye shields or tightly fitting orbital goggles, should be used to prevent damage to the eye. If unsure about safe use, request the testing specifications used by the manufacturer, to be sure that the product in question has been tested with the wavelength and within the clinical parameters to be used. If the documentation is not available, do not use the product.

Remember - Eye injury is completely preventable if everyone in the laser room is properly trained, and uses proper eye protection at all times during laser use, with no exceptions.

\section{Flammability and Reflection}

Flammability is a potential laser hazard associated with most high power systems, and many flammable products are used routinely in surgery. These items may include: dry or non-woven fabrics, plastic, rubber, solutions containing alcohol, tape removers, skin degreasers, disposables, skin preparation solutions, paper, foam positioning devices, etc.

Solutions that contain iodophors, (Hibiclens, Betadine, etc.) must be thoroughly dry, according to the stated drying times in the manufacturer's instructions for use, before firing the laser. Fire hazards occur when exposed to the heat produced by a high intensity laser beam. The risk applies to everyone in the room including the patient.

Methane gas is highly flammable, and can be present in the perianal area while a patient is in lithotomy position for a gynecologic or urologic laser procedure. If the beam interacts with this gas, a fire can occur and patients can suffer severe burns. Protection is provided by covering the area with wet sponges or towels, and ensuring good contact with all tissue folds in the area.

When patients present for procedures at the hairline, the area must be washed free of any cosmetic preparation (hair spray, gels, mousse, etc.) that may contain alcohol. This should be a standard nursing procedure to be completed at the time of pre-treatment patient admission and preparation.

An open basin of water for quenching fire in flammable materials should positioned near the laser operator, and a standard electrical equipment fire extinguisher should be quickly and easily available near the laser room

Remember - FIRE is a NEVER event in the operating room. Be vigilant - know what is in and around the laser target site at all times. Be competent and ready to respond to fire.

\section{Testing and Calibration}

Infrared lasers with coaxial visible aiming beams must be tested for alignment and for the presence of an appropriate beam mode, while fiberoptic lasers must be calibrated for adequate transmission across the fiber, in order to assure accurate and consistent delivery of energy to tissue.

Testing should be done according to manufacturer's instructions, before the first patient of the day, and then repeated 
if the laser is moved or if the delivery system is changed. The nurse or operator can test however, should there be a question of suitability, the user must make the decision as to whether or not it can be used on the patient.

\section{Electrical Hazards}

Lasers are electrical devices, and should be treated with the same caution as any other piece of electrical equipment. Standard electrical safety procedures must be followed, and an occupational health and safety plan for response to fire, should be in place and included in staff education programs.

The laser operator should examine the unit while setting up and testing, to be sure that all electrical cords, plugs, and connections are intact and in safe working condition. Extension cords should not be used on lasers, and in many cases, isolated circuits are necessary to prevent power drains in the laser treatment room.

It is important to check the cord connection if using an electrically powered footswitch, to be sure that there are no damaged or exposed wires. Improper handling of the footswitch can result in this type of damage, and if not repaired properly, can cause electrical shock upon activation.

\section{Airborne Contaminants}

As with all potential hazards, airborne contaminants are associated with only certain wavelengths and applications, and the LSO must evaluate this for each system and use, providing appropriate protection as needed.

Research has proven that thermal disruption of viable human cells, regardless of the instrument used, results in the release of toxic, mutagenic and carcinogenic materials, including carbon, intact viral particulates (including HPV, HepB, and HIV) bacteria, blood borne pathogens, and over 41 known hazardous gases such as benzene, formaldehyde, toluene, and carbon monoxide.

\section{Surgical plume is more than an inconvenience, and must be treated as an occupational hazard with a high level of risk. \\ Standards require plume evacuation systems to have ULPA filters (Ultra Low Particulate Air Filter) that remove partic- ulates to 0.1 micron, (mean average diameter of viral particulates found in plume), and have an efficiency rating of $99.999 \%$.}

HEPA (High Efficiency Particulate Air Filter) filters are NOT adequate, filtering only to particle sizes of 0.3 microns (mean average diameter of bacteria).

Masks are not meant to be the first line of protection against exposure to surgical plume. According to current research, there are no masks on the market today, including high filtration and N95 masks, that are capable of filtering out all airborne contaminants and gases. To be at all effective, N95 masks must be fit tested for each employee, according to Occupational Health and Safety procedures, to be sure of a tight seal, and must be secured and worn properly at all times for ALL plume producing procedures - about $95 \%$ of all surgical cases. This can be a costly and relatively ineffective control measure.

Wall suction lines should always be protected with in-line filters that are placed between the wall inlet and the floor canister. Though these filters may decrease suction strength, they will prevent the build-up of particulates and debris in suction lines in the wall, that often resulting in expensive repairs to the central vacuum system.

All materials used to collect and handle surgical plume, should be considered as biohazard, and disposed of according to infection control procedures. Staff must wear masks, goggles, and gloves, to change and handle filters, and should place all used materials in biohazard bags.

Remember - It is time to stop smoking in the operating room - use plume scavenging systems EVERY DAY, in EVERY ROOM, and on EVERY CASE ! 
Question four - "How do we ensure that our laser program will be sustainable, meet changing regulations, standards, and guidelines, and function at a high level of safety for all staff and the patients in their care ?"

Risk Management Step Four - Audit and quality management,

Safety audits monitor compliance with standards and facility policies and procedures. Audit should be conducted at least once per year, under the supervision of the LSO, and performed by a qualified individual who is familiar with the clinical environment, and the requirements found in standards.

The goal is to identify areas of deficiency and may include: learning needs, need for new or additional instrumentation or safety equipment, need for policy review, additional control measures, or revision of current procedures. Audits should always be done when new systems or procedures are initiated.

Audit requires completing each of the following steps:

1. Inventory all equipment and develop a checklist

2. Inspect every item on the checklist, assessing it's condition, placement, and handling

3. Interview staff working with the laser systems

4, Observe laser procedures (set up, testing, and intraoperative management)

5. Document results

6. Remedy deficiencies identified

7, Monitor outcomes and follow-up

Results should be documented and reported to the program administrator, and LSO for remedial action. Should there be an accident, incident, or occurrence, documentation of a current audit will help substantiate that the user maintained safe systems, and staff compliance, in accordance with standards and workplace safety policies.

In conclusion, it must be evident that developing a standards based safety plan is the first and most critical responsibility of a healthcare facility, and it's management team, before a laser is put into service in surgery.

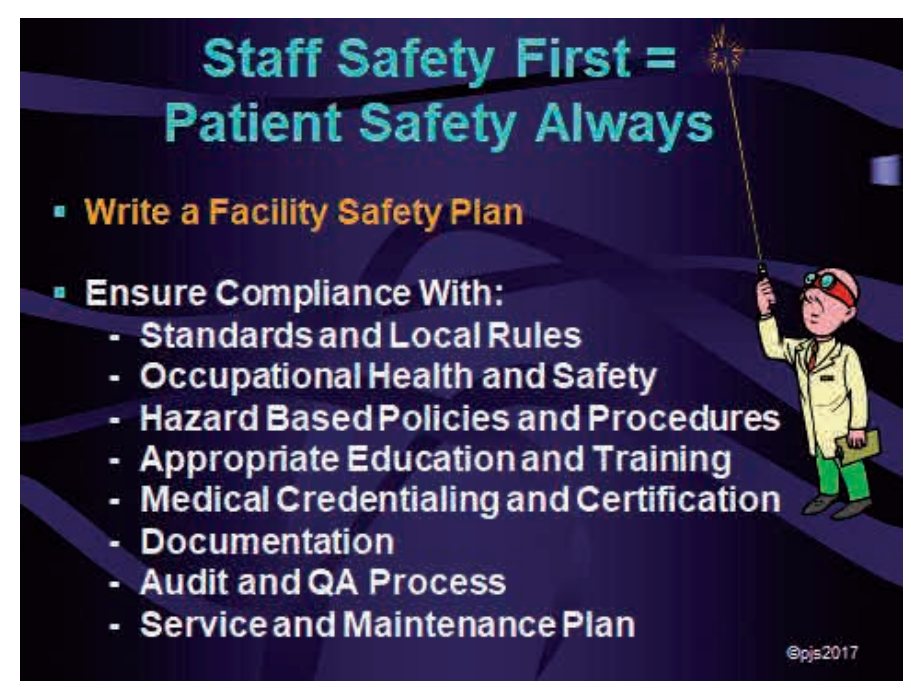

Laser team members, whether they adopt the CRM communication system or not, must be empowered through education and training, to contribute to the daily operations of the laser service, to work safely and collaboratively, and to become safety advocates for their patients.

Laser surgery presents challenges, hazards, risks, and opportunities, and safety should never be taken for granted. Commitment to compliance, constant vigilance, and continuing education and training, are the keys to safe and positive outcomes for all.

\section{Remember - laser safety is everyone's responsibility !}

